\title{
Control method of active parallax barrier and binocular image for glasses-free stereoscopic display according to viewing position
}

\section{Hiiro Nakamura, Tomoya Kitada, Goro Hamagishi, Kayo Yoshimoto, Kaoru Kusafuka, Hideya Takahashi}

\begin{tabular}{|c|l|}
\hline Citation & Proceedings Volume 11304, Advances in Display Technologies X; 1130414 \\
\hline Issue Date & 2020-02-26 \\
\hline Description & Event: SPIE OPTO, 2020, San Francisco, California, United States \\
\hline Type & Conference Paper \\
\hline Textversion & Author \\
\hline Rights & $\begin{array}{l}\text { C (2020) COPYRIGHT Society of Photo-Optical Instrumentation Engineers } \\
\text { (SPIE). One print or electronic copy may be made for personal use only. } \\
\text { publication for a fee or for commercial purposes, and modification of the contents } \\
\text { of the publication are prohibited. } \\
\text { After it is published, it will be found at https://doi.org/10.1117/12.2545394. }\end{array}$ \\
\hline DOI & 10.1117/12.2545394 \\
\hline
\end{tabular}

\author{
Self-Archiving by Author(s) \\ Placed on: Osaka City University
}

Hiiro Nakamura, Tomoya Kitada, Goro Hamagishi, Kayo Yoshimoto, Kaoru Kusafuka, and Hideya Takahashi "Control method of active parallax barrier and binocular image for glasses-free stereoscopic display according to viewing position", Proc. SPIE 11304, Advances in Display Technologies X, 1130414 (26 February 2020); https://doi.org/10.1117/12.2545394 


\title{
Control method of active parallax barrier and binocular image for glasses-free stereoscopic display according to viewing position
}

\author{
Hiiro Nakamura ${ }^{a}$, Tomoya Kitada ${ }^{a}$, Goro Hamagishi ${ }^{a}$, Kayo Yoshimoto ${ }^{a}$, Kaoru Kusafuka ${ }^{\mathrm{b}}$, \\ Hideya Takahashi ${ }^{* a}$

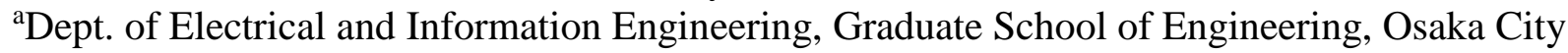 \\ Univ., 3-3-138 Sugimoto, Sumiyoshi-ku, Osaka 558-8585, Japan; \\ ${ }^{\mathrm{b}}$ Kyocera Corp., 800 Ichimiyake, Yasu 520-2362, Japan
}

\begin{abstract}
In 3D displays based on parallax barriers, active parallax barriers that can change the barrier pattern according to the viewing position have been proposed to expand the viewing area. However, the production cost increases because the active barrier requires a special LC panel. Therefore, to lower the cost, we propose a glasses-free stereoscopic display using an active parallax barrier of an LC panel with the same specifications as the image LC panel. The ideal image pattern cannot be formed because the minimum control unit of the LC panel is equal to one subpixel. However, by using an image cycle pitch method (ICPM) that periodically increases the horizontal pitch of one pair of binocular images by one subpixel, we can realize the ideal relationship between the average pitch of the binocular image and the barrier pitch. In our previous research, we could make the average pitch match to the ideal pitch on the discrete optimum viewing distances (OVDs), but we could not follow the viewing position between the discrete OVDs. In this paper, we propose the ICPM that can keep the stereoscopic vision even at any viewing position. In order to verify the effectiveness of the proposed method, we made the prototype displays and evaluated them. As a result, we showed the crosstalk ratio could be suppressed low over a very wide range by using the proposed method. Also, we confirmed we could obtain the stereoscopic vision at the viewing distance from $300 \mathrm{~mm}$ to $729 \mathrm{~mm}$ by a subjective evaluation.
\end{abstract}

Keywords: glasses-free stereoscopic display, active parallax barrier, step barrier, eye tracking, wide viewing area

\section{INTRODUCTION}

There are some demands for glasses-free 3D displays in medical, educational, and entertainment fields. One of the simplest glasses-free 3D displays is a glasses-free stereoscopic display using a parallax barrier. It has high image quality, but the viewing area that the viewer can obtain stereoscopic vision is very narrow ${ }^{1}$. Then, it is possible to expand the viewing area by using eye tracking control that tracks the eye position of the viewer using a depth camera and changes the image pattern according to the viewing position ${ }^{2-4}$. However, there is a limit to expand the viewing area just by image processing . $^{5}$ Therefore, methods using an LC panel as an active parallax barrier have been proposed ${ }^{6,7}$. The viewing area can be further expanded by changing the barrier pitch according to the viewing distance. Also, 2D/3D modes or portrait/landscape modes can be switched. However, the active parallax barrier requires a special LC panel, so the production cost increases.

In this paper, to make the cost lower, we propose a glasses-free stereoscopic display using an active parallax barrier of an LC panel with the same specifications as the image LC panel. The design of the special LC panel is not required, and the production process of the active barrier LC panel can be shared with that of the image LC panel. Therefore, we can greatly lower the cost.

*hideya@osaka-cu.ac.jp 
The ideal image pattern cannot be formed because the minimum control unit of the LC panel is equal to one subpixel. By using an image cycle pitch method (ICPM) that periodically increases the horizontal pitch of one pair of binocular images on the image LC panel by one subpixel, we can realize the ideal relationship between the average pitch of binocular image and the barrier pitch, and provide the 3D image with low crosstalk. In our previous research ${ }^{8}$, we could realize multiple discrete optimum viewing distances (OVDs) by changing the barrier pitch, and make the average pitch of binocular image match to the ideal pitch on the OVD. However, we could not follow the viewing position between the discrete OVDs. In this paper, we propose the ICPM that can keep the stereoscopic vision even at the viewing position between the discrete OVDs. Therefore, we can obtain the stereoscopic vision even if the viewer moves to any viewing position.

In chapter 2, we show the parallax barrier design method. In chapter 3, we explain the principle of the ICPM and show the viewing area expansion method using the eye tracking control. In chapter 4, we make the prototype displays and measure the crosstalk to verify the effectiveness of the proposed method. In chapter 5, we discuss the conclusion.

\section{PARALLAX BARRIER DESIGN METHOD}

This chapter shows a conventional parallax barrier design method ${ }^{9}$. Figure 1 shows a positional relationship between a slanted parallax barrier and an image display. The slanted line is the inclination angle of the parallax barrier aperture and the angle $\theta$ is given by

$$
\theta=\tan ^{-1}\left(\frac{a H_{p}}{b V_{p}}\right)
$$

where $H_{p}$ and $V_{p}$ are the horizontal and vertical pitches of the subpixel, and $a$ and $b$ are the number of the subpixels that the slanted line crosses in the horizontal and vertical directions. We form one pair of binocular images and one pair of aperture and shield areas of the parallax barrier in the cycle of $n$ subpixels along the inclination angle, and assign numbers to the subpixels from 1 to $n$. In Fig. $1, n$ is 20, and right eye images are arranged in the subpixels from \#1 to \#10 and left eye images are arranged in the subpixels from \#11 to \#20. The barrier has a step shape. Then, $B_{p}$ is the barrier pitch, $A_{w}$ is the barrier aperture width, $k$ is the horizontal pitch of one pair of binocular images, $j$ is the barrier aperture width projected on the image display, and $g$ is the distance between the parallax barrier and the image display. In the barrier LC panel, the aperture can be realized by displaying white images.

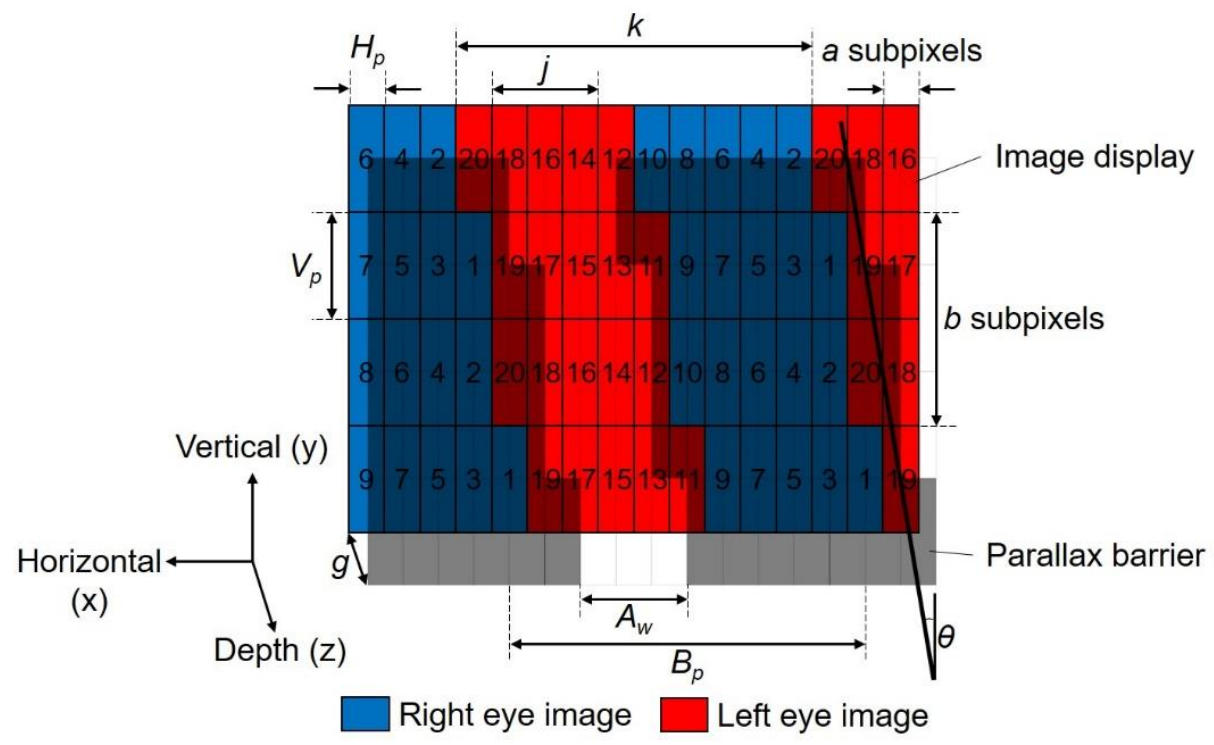

Figure 1. Positional relationship between slanted parallax barrier and image display. $H_{p}$ and $V_{p}$ are the horizontal and vertical pitches of the subpixel, $a$ and $b$ are the number of the subpixels that the slanted line crosses in the horizontal and vertical directions, $B_{p}$ is the barrier pitch, $A_{w}$ is the barrier aperture width, $k$ is the horizontal pitch of one pair of binocular images, $j$ is the barrier aperture width projected on the image display, and $g$ is the distance between the parallax barrier and the image display. 
Figure 2 shows a positional relationship between a viewer, a parallax barrier, and an image display. From the geometric relationship in Fig. 2, Eqs. (2), (3), and (4) are given by

$$
\begin{gathered}
E: d=\frac{k}{2}: g, \\
d: B_{p}=(d+g): k,
\end{gathered}
$$

and

$$
d: A_{w}=(d+g): j,
$$

where $E$ is the interocular distance and $d$ is the optimum viewing distance. The image LC panel and the barrier LC panel are the same, so $B_{p}$ and $k$ need to be an integral multiple of $H_{p} / b$. However, in Eq. (3), $k>B_{p}$ holds due to $g>0$. Therefore, if $B_{p}$ is an integral multiple of $H_{p} / b, k$ is not. This problem is described in Chapter 3.

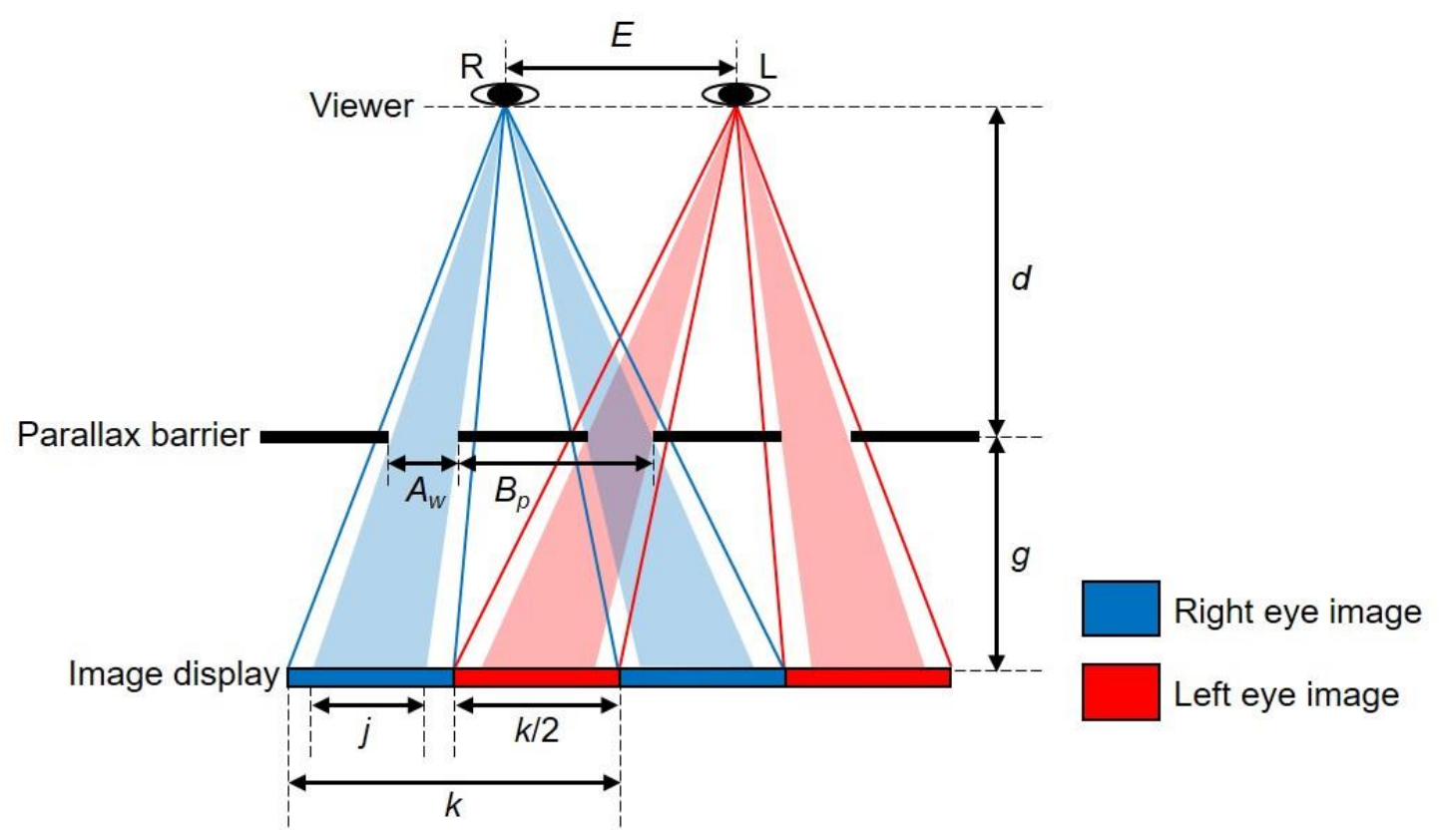

Figure 2. Positional relationship between viewer, parallax barrier, and image display. $E$ is the interocular distance and $d$ is the optimum viewing distance.

\section{IMAGE CYCLE PITCH METHOD}

\subsection{Previous image cycle pitch method (ICPM)}

As described in Chapter 2, $k$ is not an integral multiple of $H_{p} / b$ and the ideal binocular image pitch cannot be formed. Therefore, crosstalk occurs. In order to suppress the crosstalk, by increasing $k$ by one subpixel in every period $C_{p}$, we can provide the 3D image with low crosstalk. We call the period $C_{p}$ "image cycle pitch", and the above method "image cycle pitch method (ICPM)". Figure 3 shows the principle of the image cycle pitch method. In Fig. 3(a), crosstalk occurs due to the accumulated error for the ideal pitch, but the crosstalk is suppressed by increasing $k$ by $H_{p} / b$ in every $C_{p}$ in Fig. 3(b).

In our previous research, $C_{p}$ was given by

$$
C_{p}=\frac{k H_{p}}{b\left(k-B_{p}\right)}
$$

From Eq. (3), $k$ and $B_{p}$ were eliminated. However, we could not follow the viewing position between the discrete OVDs because Eq. (3) holds only at the OVD. Therefore, the viewing area was limited. 


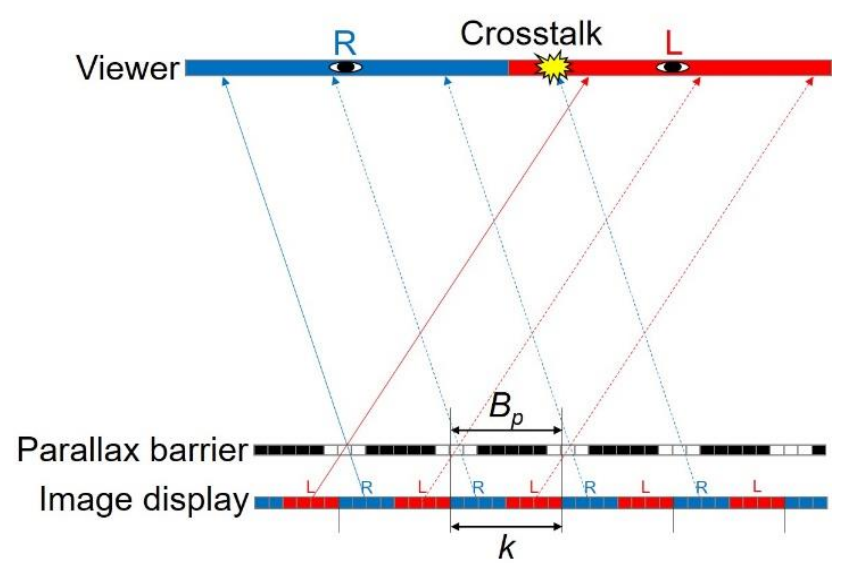

(a)

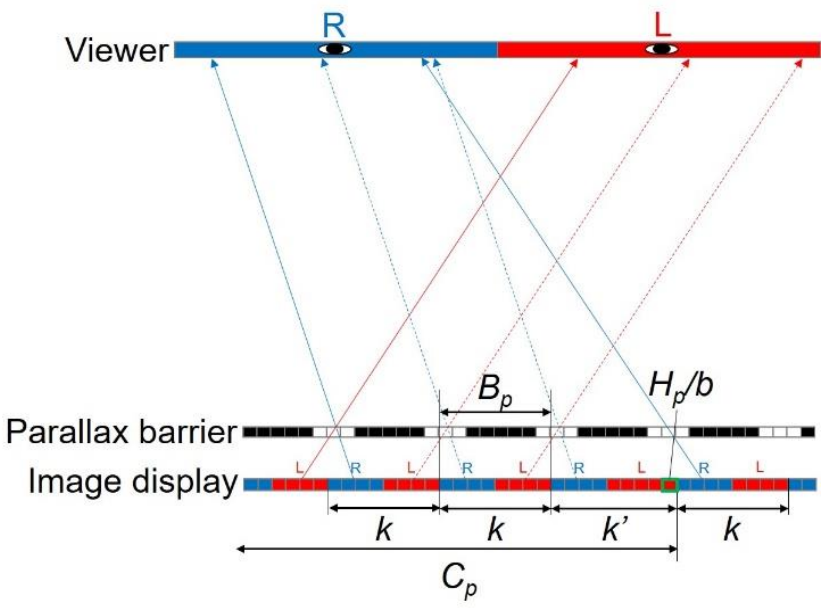

(b)

Figure 3. Principle of image cycle pitch method (ICPM). $C_{p}$ is the image cycle pitch. The crosstalk is suppressed by increasing $k$ by $H_{p} / b$ in every $C_{p}$. (a) Not ICPM. (b) ICPM. $k$ ' is $k+H_{p} / b$.

\subsection{ICPM for viewing position between discrete OVDs}

In this section, we describe the ICPM that can keep the stereoscopic vision even at the viewing position between the discrete OVDs. Figure 4 shows a relationship between an image cycle pitch $C_{p}$ and a viewing distance $z$. From Fig. 4, Eqs. $(6),(7)$, and (8) are given by

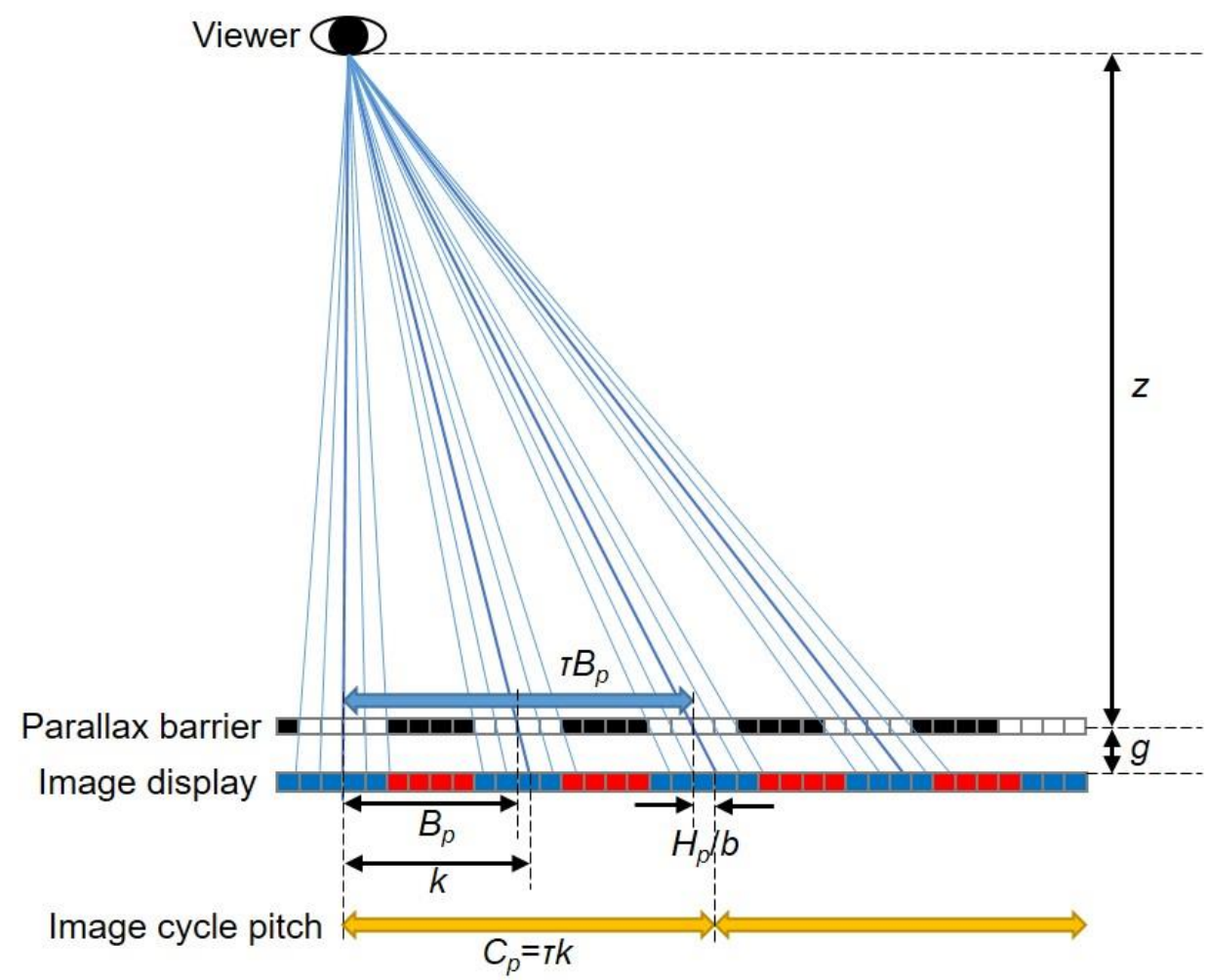

Figure 4. Relationship between image cycle pitch and viewing distance. $z$ is the viewing distance. $C_{p}$ is proportional to $\mathrm{z}$. 


$$
\begin{gathered}
C_{p}=\tau k \\
C_{p}=\tau B_{p}+\frac{H_{p}}{b},
\end{gathered}
$$

and

$$
z: B_{p}=(z+g): k,
$$

where $\tau$ is a natural number. From Eqs. (6), (7), and (8), $C_{p}$ is given by

$$
C_{p}(z)=\frac{H_{p}}{b g} z+\frac{H_{p}}{b} .
$$

From Eq. (9), $C_{p}$ is proportional to $z$.

Figure 5 shows distribution of patterns composing binocular images formed on the image display. The width of the same pattern area is equal to $C_{p}$. In Fig. 5, the subpixels that compose the binocular images shift by one subpixel when the pattern number changes by one. For example, in the pattern 1, the right eye images are arranged in the subpixels from \#1 to \#10 and the left eye images are arranged in the subpixels from \#11 to \#20, and in the pattern 2, the right eye images are arranged in the subpixels from \#2 to \#11 and the left eye images are arranged in the subpixels of \#1 and from \#12 to \#20. Then, $k$ increases by $H_{p} / b$ and the average pitch of the binocular image in the same pattern area becomes ideal. As shown in Fig. 5(a), by forming the pattern area in every $C_{p}$ centered on the front of the interocular position, we can suppress the crosstalk on the entire display. Fig. 5(b) shows the pattern distribution on the image display observed from the viewer. The border of the pattern area is along the inclination angle of the barrier aperture.

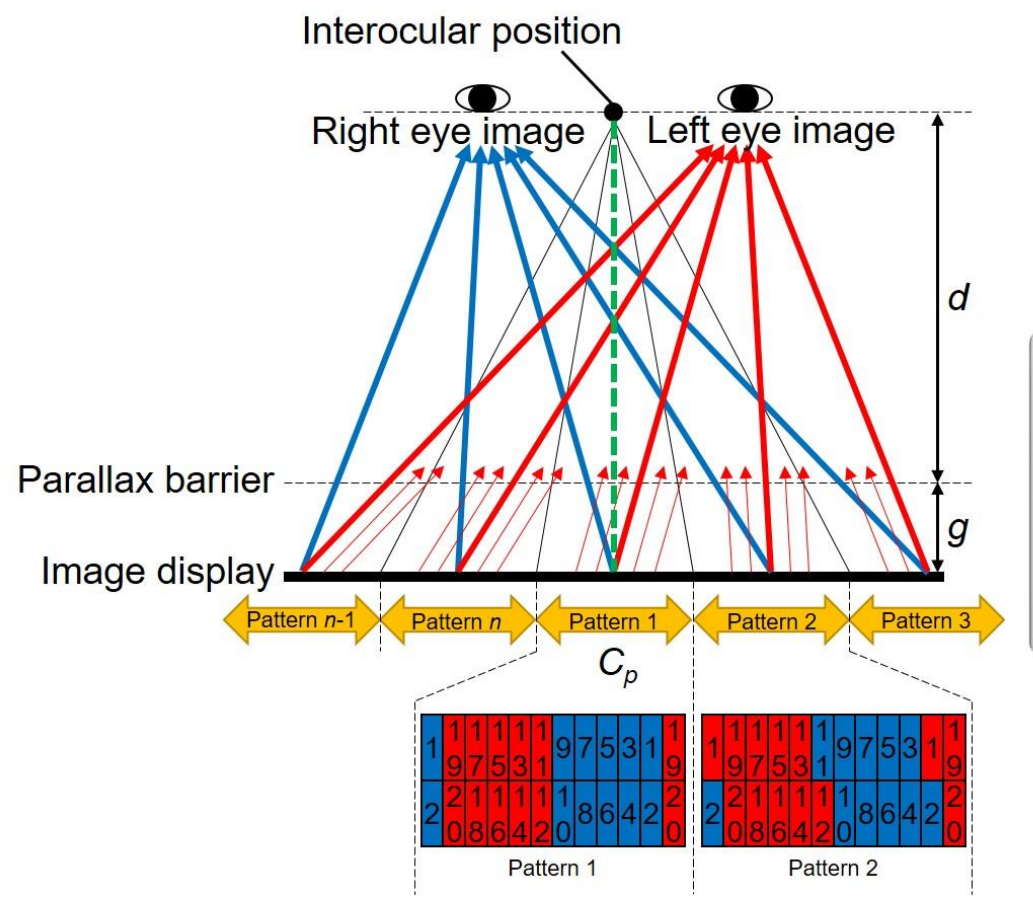

(a)

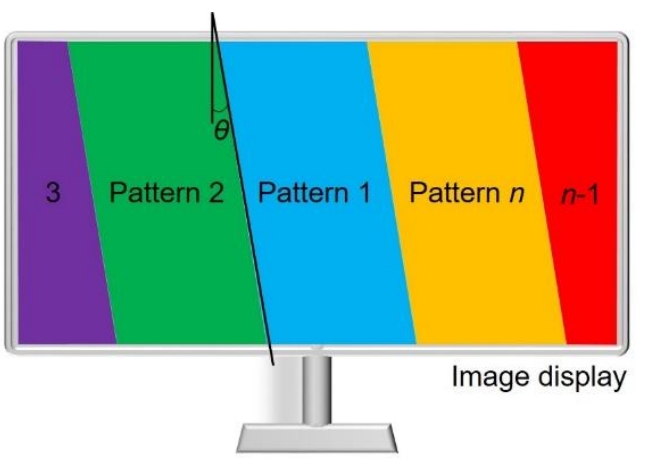

(b)

Figure 5. Distribution of patterns composing binocular images. (a) Forming pattern distribution. (b) Pattern distribution observed from viewer.

\subsection{Expansion method of viewing area using eye tracking control}

In glasses-free stereoscopic displays, crosstalk occurs when the viewer moves from the optimum viewing position. Therefore, we suppress the crosstalk by eye tracking control that tracks the eye position of the viewer using a depth camera and changes the pattern of the image and the barrier according to the position. 
Consider the case where the viewer moves from the optimum viewing position on the OVD. The OVD can be changed by changing the barrier pitch. Figure 6 shows the distribution of the patterns composing the binocular images when the viewer moves from the position shown in Fig. 5. As shown in Fig. 6(a), when the viewer moves in the horizontal direction, the crosstalk can be suppressed by shifting the pattern area according to the movement of the viewer. As shown in Fig. 6(b), when the viewer moves in the depth direction, the crosstalk can be similarly suppressed by changing the width of the pattern area according to the viewing distance.

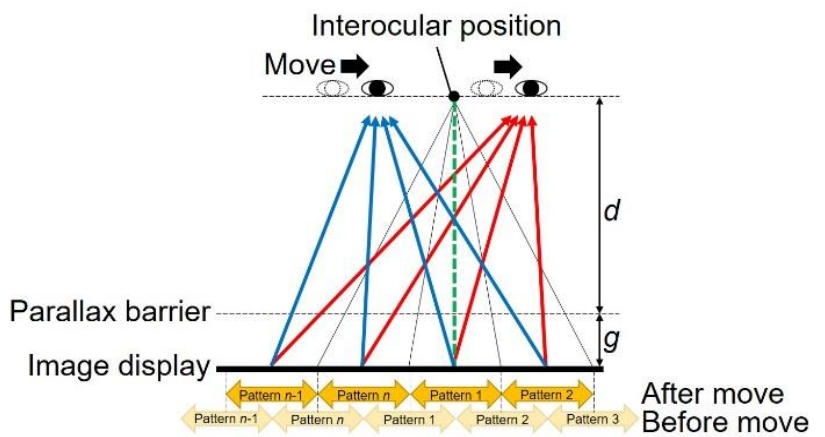

(a)

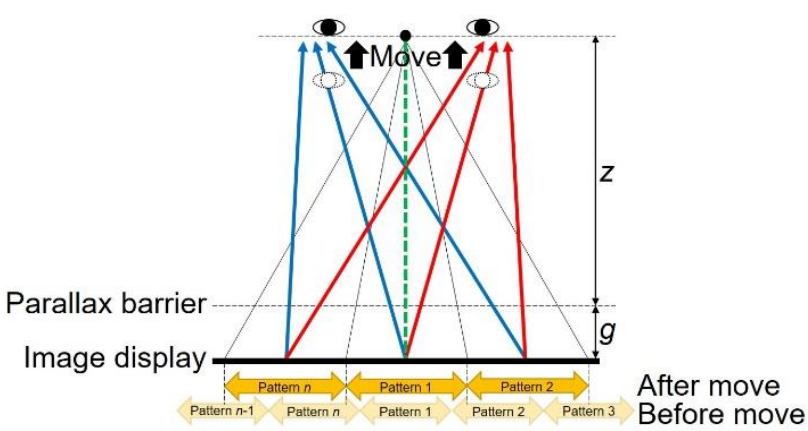

(b)

Figure 6. Expansion method of viewing area using eye tracking control. The crosstalk can be suppressed by eye tracking control that tracks the eye position of the viewer using a depth camera and changes the image pattern according to the position. (a) Movement in horizontal direction. (b) Movement in depth direction.

When the viewer moves from the OVD, subpixels observed simultaneously with the right and left eyes appear ${ }^{10}$. In this paper, all the subpixels were processed as right eye images considering human dominant eyes.

\section{EXPERIMENTAL RESULT}

\subsection{Prototype display}

In order to verify the effectiveness of the proposed method, we made two prototype displays and evaluated them. Figure 7 shows the structure of the prototype display and Figure 8 shows the appearance of the display, respectively. The prototype display was composed of a parallax barrier and an LC panel. The parallax barrier was a step barrier and the same specifications as the image LC panel. Table 1 shows the specifications of the prototype displays. The barrier pitch of the prototype display A was $0.3 \mathrm{~mm}$ and that of the prototype display B was $0.6 \mathrm{~mm}$. The aperture ratio is $A_{w} / B_{p}$, and that of both displays was $30 \%$. Also, the inclination angle of those was $\tan ^{-1}(1 / 6)$.

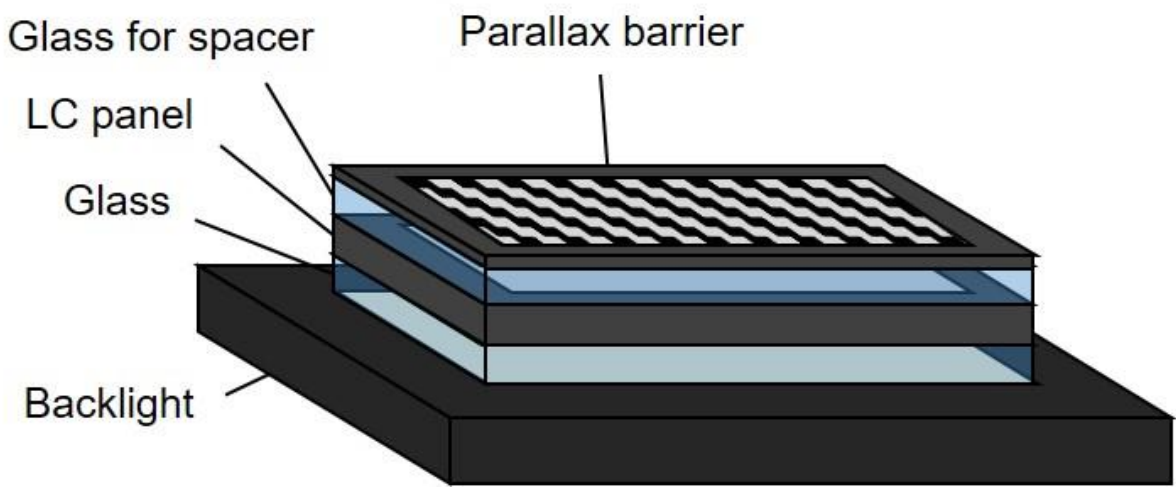

Figure 7. Structure of prototype display. The prototype display was composed of a parallax barrier and an LC panel. 


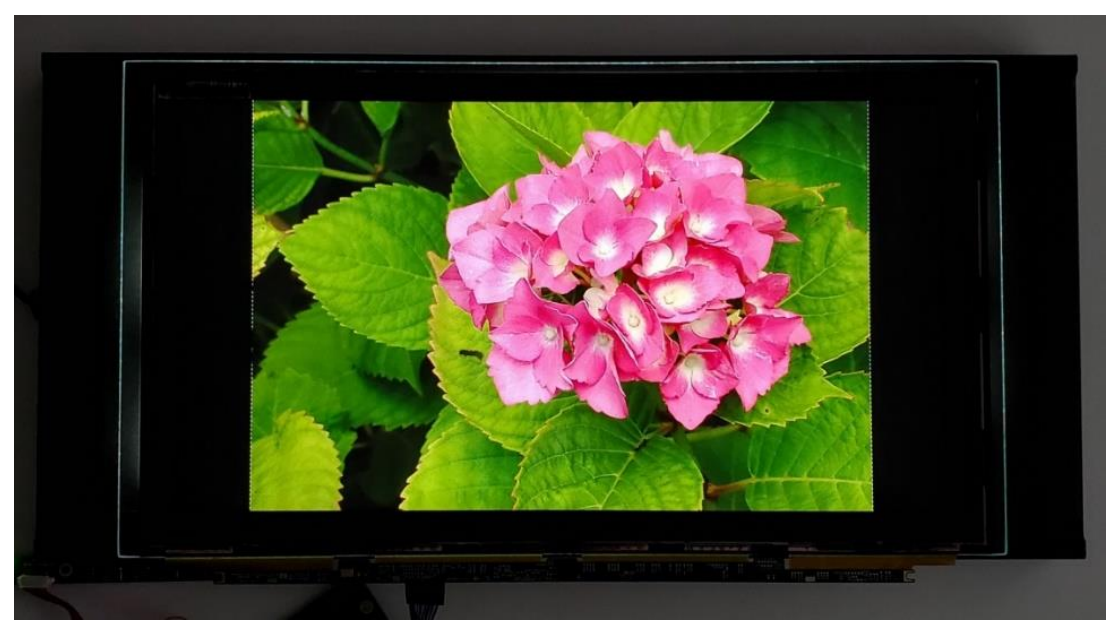

Figure 8. Appearance of prototype display. The OVDs of the prototype display A and B were $689 \mathrm{~mm}$ and $344 \mathrm{~mm}$, respectively.

Table 1. Specifications of prototype display A and B.

\begin{tabular}{|c|c|c|}
\hline & Prototype display A & Prototype display B \\
\hline Display resolution $\mathrm{H} \mathrm{x} \mathrm{V}$ & \multicolumn{2}{|c|}{3840 px x 2160 px } \\
\hline Subpixel size $H_{p} \times V_{p}$ & \multicolumn{2}{|c|}{$0.03 \mathrm{~mm} \times 0.09 \mathrm{~mm}$} \\
\hline Interocular distance $E$ & \multicolumn{2}{|c|}{$63 \mathrm{~mm}$} \\
\hline Distance between LC panel and parallax barrier $g$ & \multicolumn{2}{|c|}{$1.645 \mathrm{~mm}$} \\
\hline Inclination angle $\theta$ & \multicolumn{2}{|c|}{$\tan ^{-1}(1 / 6)$} \\
\hline Barrier pitch $B_{p}$ & $0.3 \mathrm{~mm}$ (10 subpixels) & $0.6 \mathrm{~mm}$ (20 subpixels) \\
\hline Aperture width $A_{w}$ & $0.09 \mathrm{~mm}$ (3 subpixels) & $0.18 \mathrm{~mm}$ (6 subpixels) \\
\hline Aperture ratio & \multicolumn{2}{|c|}{$30 \%$} \\
\hline Subpixel group $n$ & 20 subpixels & 40 subpixels \\
\hline Optimum viewing distance $d$ & $689.237 \mathrm{~mm}$ & $343.796 \mathrm{~mm}$ \\
\hline
\end{tabular}

\subsection{Evaluation of crosstalk}

We measured the crosstalk ratio for the right and left eyes at each viewing distance using the prototype displays. Figure 9 shows the appearance of the experimental system. In the experiment, we used a prototype display, a camera, and a moving stage. In order to measure the crosstalk ratio, we captured black images, white images, and black-and-white images from each viewing distance. The black-and-white image is an image synthesized with the right eye image as a black image and the left eye image as a white image in the case measuring the crosstalk for the right eye. We calculated the average pixel value for each captured image by image processing. Then, the crosstalk ratio $C_{r}$ is given by

$$
C_{r}=\frac{P_{v c}-P_{v b}}{P_{v w}-P_{v b}} \times 100
$$

where $P_{v b}$ is the average pixel value of the black image, $P_{v w}$ is that of the white image, and $P_{v c}$ is that of the black-andwhite image. Figure 10 shows the theoretical values of the crosstalk ratio and Figure 11 shows the measurement results. The measurement range in the depth direction was from $490 \mathrm{~mm}$ to $729 \mathrm{~mm}$ in the prototype display A and from $300 \mathrm{~mm}$ to $549 \mathrm{~mm}$ in the prototype display B. The vertical axis shows the crosstalk ratio and the horizontal axis shows the viewing distance. In the case of the proposed method, the black-and-white images were changed according to the viewing position, 
but in the case of the previous method, the images for the discrete OVDs were displayed at all the measurement distances. The green line shows the result of the prototype display A using the proposed method, the blue line shows that of the prototype display A using the previous method, the yellow line shows that of the prototype display B using the proposed method, and the red line shows that of the prototype display B using the previous method. Also, the dotted line shows the crosstalk ratio for the right eye and the solid line shows that for the left eye.

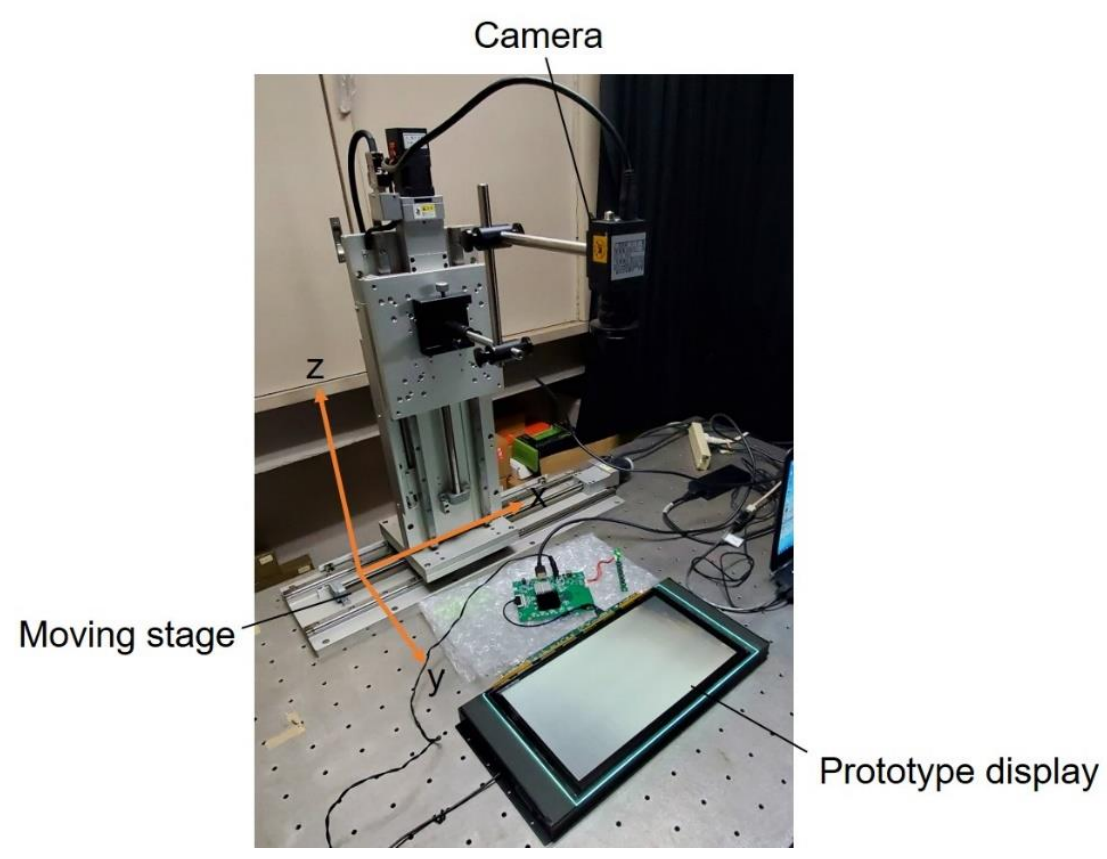

Figure 9. Appearance of experimental system. We used a prototype display, a camera, and a moving stage.

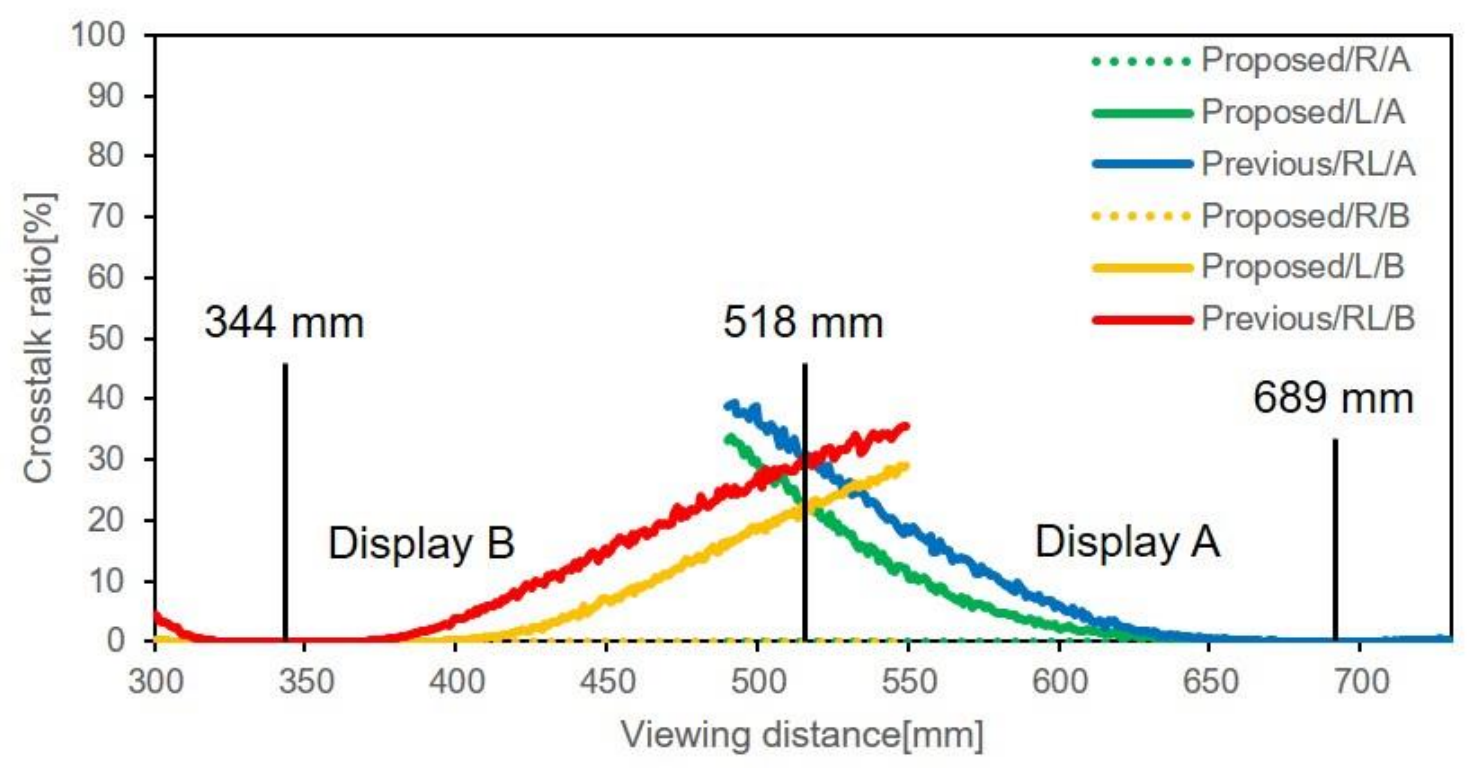

Figure 10. Theoretical values of crosstalk ratio. The vertical axis shows the crosstalk ratio and the horizontal axis shows the viewing distance. All the subpixels observed simultaneously with both eyes were processed as the right eye images, so the crosstalk ratio for the right eye was low at any viewing distance in the proposed method. 


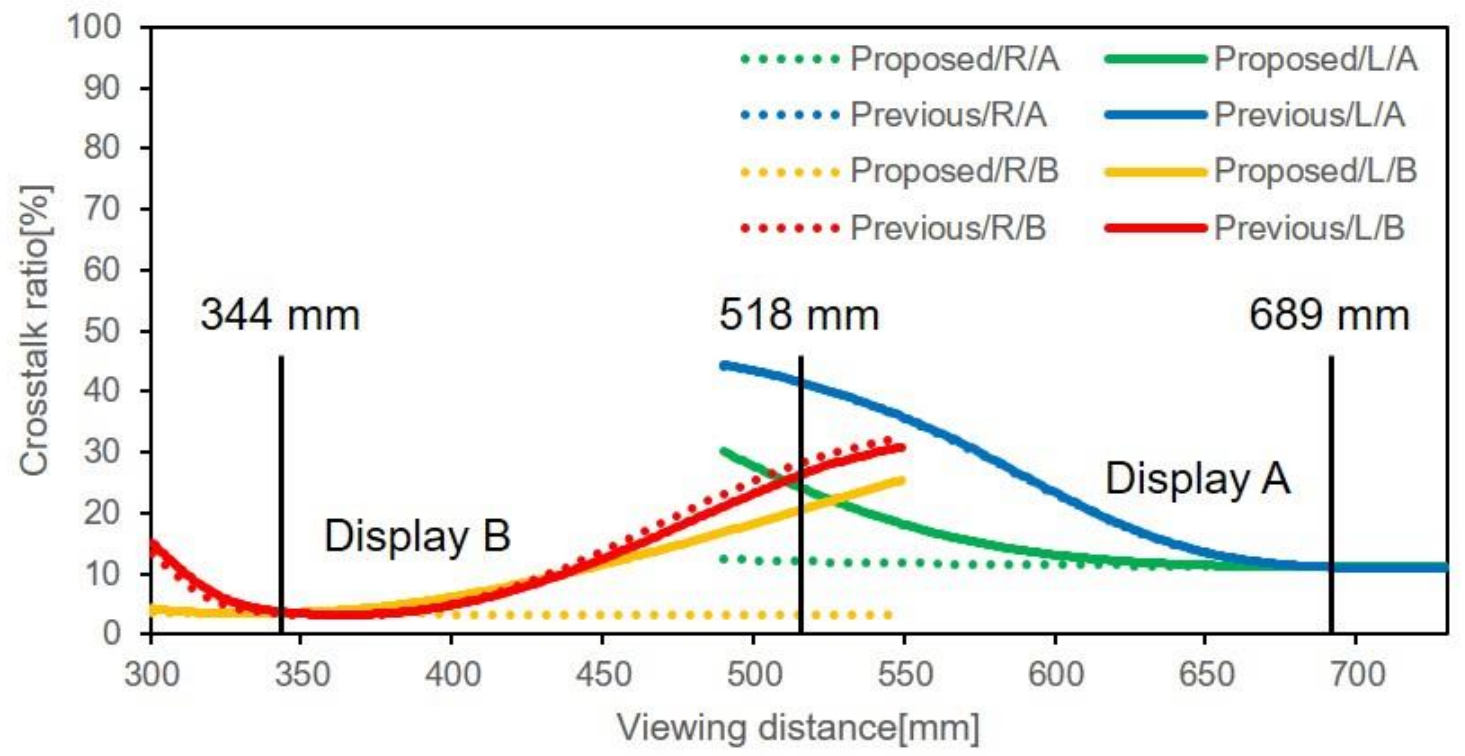

Figure 11. Measurement results of crosstalk ratio. The measurement range in the depth direction was from $490 \mathrm{~mm}$ to 729 $\mathrm{mm}$ in the prototype display A and from $300 \mathrm{~mm}$ to $549 \mathrm{~mm}$ in the prototype display B. The green line shows the result of the prototype display A using the proposed method, the blue line shows that of the prototype display A using the previous method, the yellow line shows that of the prototype display B using the proposed method, and the red line shows that of the prototype display B using the previous method. The dotted line shows the crosstalk ratio for the right eye and the solid line shows that for the left eye.

\subsection{Discussion}

In the previous method, we could not follow the viewing position between the discrete OVDs because the images for the discrete OVDs were displayed even at any distance. As a result, the crosstalk ratio increased with moving away from the discrete OVDs. On the other hand, in the proposed method, the images were changed according to the viewing position and all the subpixels observed simultaneously with both eyes were processed as the right eye images. Therefore, the crosstalk ratio for the right eye was low at any viewing distance. The crosstalk ratio for the left eye increased with moving away from the discrete OVDs, but was smaller than that of the previous method.

As the cause that the measurement results were larger than the theoretical values, the misalignment of the experimental system, the positional shift of the parallax barrier, the deflection of the display, and the light diffraction are considered. When observed from the OVD in the prototype display A, the crosstalk ratio increases by about $6.1 \%$ if the parallax barrier is shifted in the horizontal direction by one $H_{p}(0.03 \mathrm{~mm})$. In this way, even a slight misalignment that cannot be controlled by human hands increased the crosstalk. Also, in the prototype display A, the barrier aperture was very narrow and the effect of the light diffraction was large, so the crosstalk increased in particular.

We also did a subjective evaluation. In the subjective evaluation, we used a blue-and-red image synthesized with the right eye image as a blue image and the left eye image as a red image. Figure 12 shows the results of the subjective evaluation using the prototype display A and Figure 13 shows those using the prototype display B. In Fig. 12, the blue-and-red image using the proposed method was separated by the right and left eyes at the viewing distance from $518 \mathrm{~mm}$ to $729 \mathrm{~mm}$. On the other hand, in the case of the previous method, the right eye image and the left eye image were clearly mixed at the viewing distance of $518 \mathrm{~mm}$. Also, in Fig. 13, the blue-and-red image using the proposed method was separated by the right and left eyes at the viewing distance from $300 \mathrm{~mm}$ to $518 \mathrm{~mm}$. On the other hand, in the case of the previous method, the right eye image and the left eye image were clearly mixed at the viewing distance of $518 \mathrm{~mm}$. Therefore, it was obvious that the crosstalk can be suppressed low by using the proposed method at the viewing distance from $300 \mathrm{~mm}$ to $729 \mathrm{~mm}$. From the above, it can be said that the proposed method is effective. 


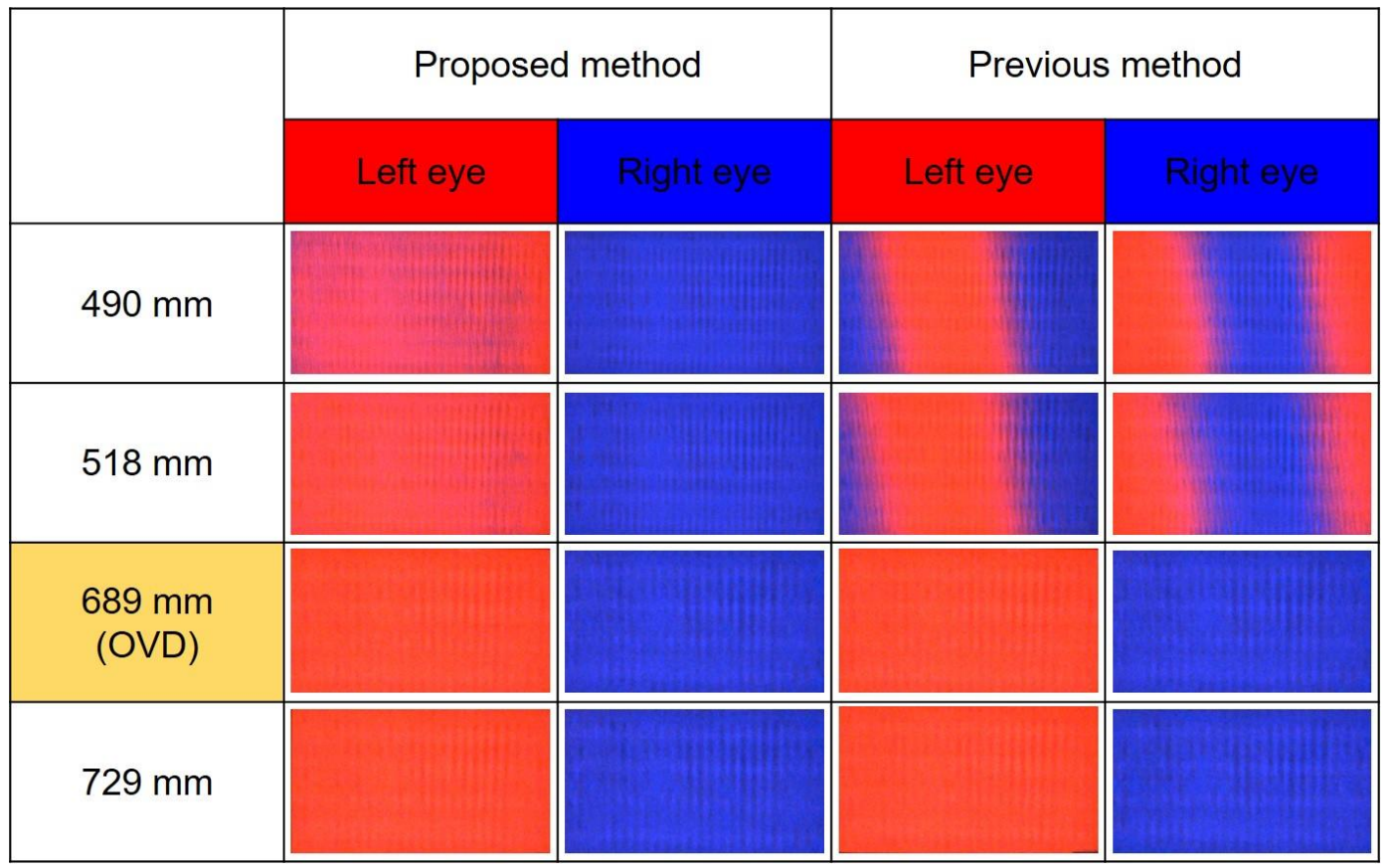

Figure 12. Results of subjective evaluation using prototype display A. The blue-and-red image using the proposed method was separated by the right and left eyes at the viewing distance from $518 \mathrm{~mm}$ to $729 \mathrm{~mm}$. On the other hand, in the case of the previous method, the right eye image and the left eye image were clearly mixed at the viewing distance of $518 \mathrm{~mm}$.

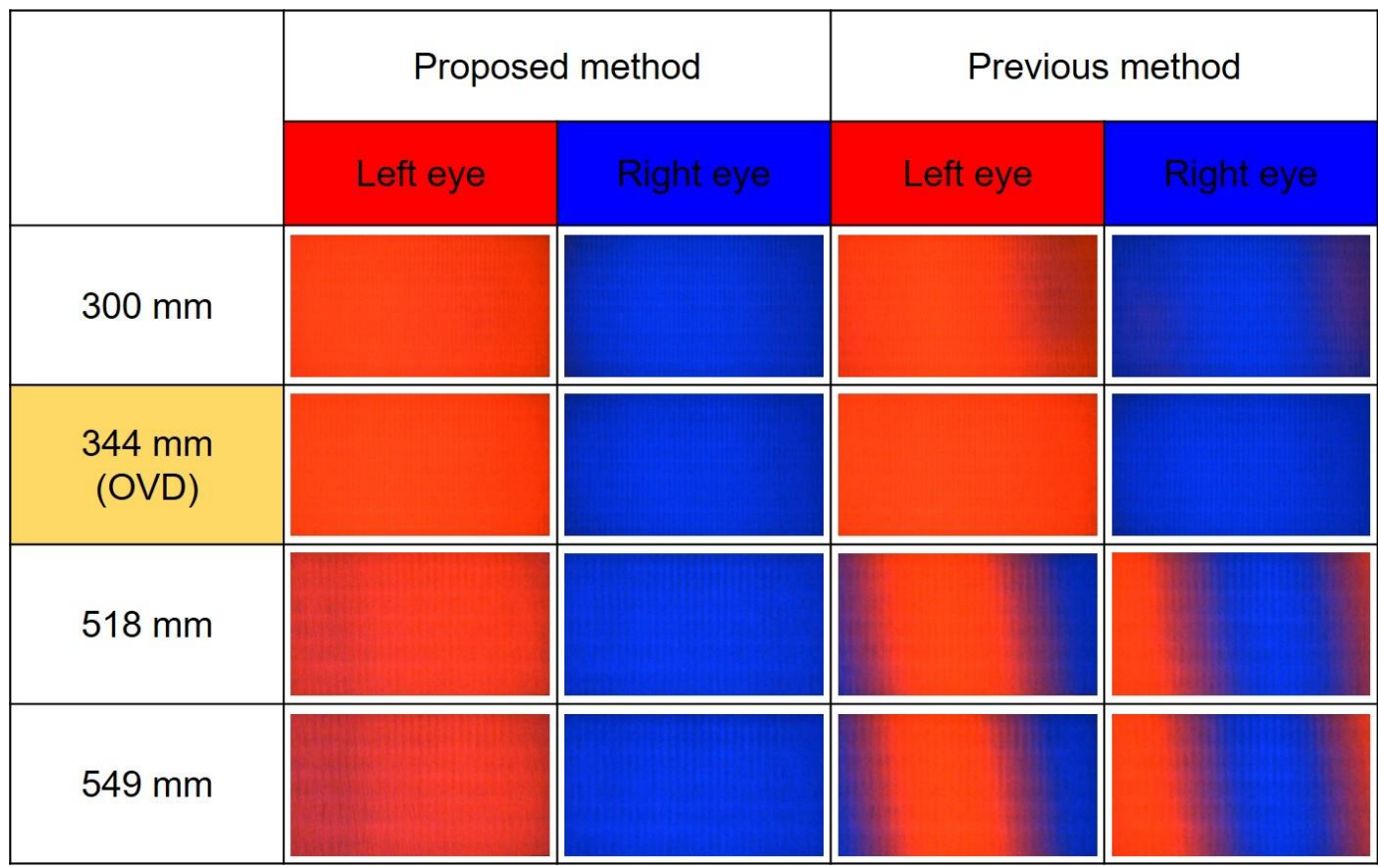

Figure 13. Results of subjective evaluation using prototype display B. The blue-and-red image using the proposed method was separated by the right and left eyes at the viewing distance from $300 \mathrm{~mm}$ to $518 \mathrm{~mm}$. On the other hand, in the case of the previous method, the right eye image and the left eye image were clearly mixed at the viewing distance of $518 \mathrm{~mm}$. 


\section{CONCLUSION}

In this paper, we proposed the glasses-free stereoscopic display using the active parallax barrier of the LC panel with the same specifications as the image LC panel. The ideal image pattern cannot be formed because the minimum control unit of the LC panel is equal to one subpixel. However, by using the ICPM that periodically increases the horizontal pitch of one pair of binocular images on the image LC panel by one subpixel, we can realize the ideal relationship between the average pitch of the binocular image and the barrier pitch. Also, we can expand the viewing area by using the eye tracking control that tracks the eye position of the viewer and changes the pattern of the image and the barrier according to the viewing position. In our previous research, we could observe the 3D image only at the discrete OVDs, but in the proposed method, we can obtain the stereoscopic vision at any viewing position between the discrete OVDs. In order to verify the effectiveness of the proposed method, we made the prototype displays and evaluated them. As a result, the crosstalk ratio could be suppressed low over a very wide range. Also, we confirmed that the right and left images were separated at the viewing distance from $300 \mathrm{~mm}$ to $729 \mathrm{~mm}$ by the subjective evaluation, and we showed the effectiveness of the proposed method. The glasses-free 3D display proposed in this paper can be realized at very low costs, so it is expected to contribute to spread 3D displays in the market.

\section{REFERENCES}

[1] Jason, G., "Three-dimensional display technologies," Advances in Optics and Photonics 5(4), 456-535 (2013).

[2] Kim, S. K., Yoon, K. H., Yoon, S. K. and Ju, H., "Parallax barrier engineering for image quality improvement in an autostereoscopic 3D display," Optics Express 23(10), 13230-13244 (2015).

[3] Yoon, K. H., Kang, M. K., Lee, H. and Kim, S. K., "Autostereoscopic 3D display system with dynamic fusion of the viewing zone under eye tracking: principles, setup, and evaluation," Applied Optics 57(1), A101-A117 (2018).

[4] Lee, S., Park, J., Heo, J., Kang, B., Kang, D., Hwang, H., Lee, J., Choi, Y., Choi, K. and Nam, D, "Autostereoscopic 3D display using directional subpixel rendering," Optics Express 26(16), 20233-20247 (2018).

[5] Nakamura, H., Hamagishi, G., Yoshimoto, K., Takahashi, H., Matsumoto, T. and Kusafuka, K., "A Novel Eye Tracking System to Expand Viewing Area in all Directions for Glasses-Free 3D Display Displayable in both Portrait and Landscape Modes," Proc. IDW'18, 792-795 (2018).

[6] Suzuki, D., Hayashi, S., Hyodo, Y., Oka, S., Koito, T. and Sugiyama, H., " A wide view auto-stereoscopic 3D display with an eye-tracking system for enhanced horizontal viewing position and viewing distance," SID Int. Symp. Digest Tech. Papers 24(11), 657-668 (2016).

[7] Fujishima, T., Nakamura, H., Tsujino, T., Hamagishi, G., Yoshimoto, K., Takahashi, H., Matsumoto, T. and Kusafuka, K., "A novel control method of the combination of simple active barrier pitch control and image processing to extremely expand the viewing zone in forward and backward directions of stereoscopic 3D displays," Proc. SPIE 10942, Advances in Display Technologies IX, 109420M (2019).

[8] Tsujino, T., Nakamura, H., Fujishima, T., Hamagishi, G., Yoshimoto, K., Takahashi, H., Matsumoto, T. and Kusafuka, K., "3D display with active parallax barrier using the monochromatic LC panel of specifications same as the image display panel," Proc. SPIE 10942, Advances in Display Technologies IX, 1094211 (2019).

[9] Minami, Y., Hamagishi, G., Yoshimoto, K. and Takahashi, H., "Portrait and landscape mode convertible stereoscopic display using parallax barrier," Proc. IS\&T Electronic Imaging, Stereoscopic Displays and Applications XXVIII, 106-112 (2017).

[10] Yamaguchi, Y., Nakamura, H., Hamagishi, G., Yoshimoto, K., Matsumoto, T., Kusafuka, K. and Takahashi, H., "Further Crosstalk Reduction Method with Eye-Tracking for Glasses-Free Stereoscopic Display in Both Portrait and Landscape Modes," Proc. IDW'19, 982-985 (2019). 